Relations industrielles

Industrial Relations

\title{
La loi des différents ouvriers de Québec (1901-1909)
}

\section{Roger Chartier}

Volume 17, numéro 2, avril 1962

URI : https://id.erudit.org/iderudit/1021633ar

DOI : https://doi.org/10.7202/1021633ar

Aller au sommaire du numéro

\section{Éditeur(s)}

Département des relations industrielles de l'Université Laval

ISSN

0034-379X (imprimé)

1703-8138 (numérique)

Découvrir la revue

Citer cet article

Chartier, R. (1962). La loi des différents ouvriers de Québec (1901-1909).

Relations industrielles / Industrial Relations, 17(2), 159-168.

https://doi.org/10.7202/1021633ar
Résumé de l'article

Voici le second d'une série de textes sur la législation québécoise du travail (et sur les services connexes des ministères pertinents) de 1885 à nos jours.
Tous droits réservés @ C Département des relations industrielles de l’Université Laval, 1962
Ce document est protégé par la loi sur le droit d'auteur. L’utilisation des services d'Érudit (y compris la reproduction) est assujettie à sa politique d'utilisation que vous pouvez consulter en ligne.

https://apropos.erudit.org/fr/usagers/politique-dutilisation/ 
Contribution à l'histoire de la législation

québécoise du travail: II

\section{La loi des différents ouvriers de Québec (1901-1909)}

\section{Roger Chartier}

Voici le second d'une série de textes sur la législation québécoise du travail (et sur les services connexes des ministères pertinents) de 1885 à nos jours.

La Loi des différends ouvriers de Québec (1 Ed. VII, ch. 31) sanctionnée le 28 mars 1901 a été la première en date de nos lois «de conciliation et d'arbitrage pour régler les différends industriels ». Des événements l'ont précédée, qui ont provoqué sa passation. Nous les analyserons immédiatement, après quoi nous ferons une étude critique de la loi elle-même et une description de son application.

\section{LA LOI FÉdÉRALE DE CONCILIATION, 1900}

L'adoption de la Loi de la conciliation («Conciliation Act») marque l'entrée du Parlement fédéral dans le domaine de la législation du travail et de la conciliation des différends du travail. En 1899, le gouvernement fédéral nomma une commission chargée d'enquêter sur les conditions de travail dans les mines de métaux de la Colombie britannique. Les enquêteurs recommandèrent au Parlement fédéral de fournir les services d'un médiateur dans les conflits de travail et de mettre sur pied un ministère du Travail. Déjà en 1889, une autre commission avait suggéré la création d'un tel ministère. L'enquête de King, dont nous avons parlé plus haut, et son rapport en 1898, mirent à jour des conditions déplorables qui amenèrent l'adoption par le Parlement, en

CHARTIER, Roger, M.Sc.Soc., professeur au Département des relations industrielles de la Faculté des sciences sociales de l'Université Laval, études graduées pour l'obtention du doctorat en sociologie à l'Université de Chicago.

- Cet article, ainsi que les autres de la série, sont la synthèse d'une « Etude analytique et évolutive des services du Ministère du travail et de la législation ouvrière et sociale de la province de Québec, 1885-1952 》 préparée par l'auteur à la demande du Ministère, et reproduite ici avec sa permission. 
1900 , d'une « Résolution concernant les justes salaires » dans les contrats du gouvernement. Le flot d'immigrants et l'industrialisation toujours croissante contribuèrent à mettre en évidence les problèmes du travail. Ainsi, la Loi de conciliation de 1900 (18 juillet) fut adoptée, et le Ministère du travail organisé pour appliquer la loi et la nouvelle politique des justes salaires. La nouvelle loi, à l'instar de la loi anglaise de 1896, pourvoyait au règlement et à la prévention des conflits de travail par le recours volontaire à un mécanisme de conciliation et d'arbitrage. Elle ordonnait en outre la publication de la Gazette du travail, "afin de répandre des données statistiques et autres renseignements exacts au sujet des conditions de la main-d'oeuvre ». Mettant sur pied le Ministère du travail, elle lui enjoignait de faire au Parlement un rapport annuel. Le premier numéro de la Gazette parut dès le mois de septembre 1900; c'était l'oeuvre de W. L. M. King.

Comme la loi anglaise de 1896 dont elle est une fidèle reproduction, la loi canadienne ne restreint en rien la liberté des patrons et des ouvriers; elle ne peut aider à la solution des conflits ouvriers que si les deux parties acceptent la conciliation et en font la demande.

\section{LA GRÈVE DE LA CHAUSSURE A Qú́BEC}

Déjà en 1881 , la ville de Québec était le centre où l'industrie des cuirs - tannerie et cordonnerie - s'exploitait le plus en grand au Canada. Le syndicalisme s'y était vigoureusement implanté. Un fabricant de chaussures, ayant renvoyé l'un de ses employés, se vit aux prises avec une grève de ses 80 employés. Vingt-deux fabricants sur 26 à Québec, nouvellement groupés en association, résolurent en fin d'octobre 1900 de fermer leurs portes de concert: 3,850 travailleurs - dont 1,235 femmes - se trouvèrent ainsi sans travail.

Comme s'exprimaient les patrons en contre-grève dans un *communiqué 》 au journal $L^{\prime}$ « Evénement » (27 octobre 1900):

La question se résume à savoir si les ouvriers continueront à gérer les manufactures à leur gré, ou si les patrons, qui ont leurs capitaux investis dans l'industrie, auront le droit d'administrer leurs ateliers à leur goût et d'employer les ouvriers qui leur conviennent, qu'ils soient ou non membres des unions... La cause des troubles est due aux exigences exagérées des ouvriers unionistes.

A mots couverts, c'était là poser la question du droit d'association et d'action syndicale. Ainsi qu'on peut le lire dans la Gazette du travail de décembre 1900:

Il fut généralement compris que le lock-out était dirigé contre les unions auxquelles les employés appartenaient, et qu'il avait pour objet de forcer les employés à abandonner toutes relations avec leurs organisations (p. 160).

L'arrêt de travail se prolongea jusqu'au 10 décembre, soit durant six semaines. Finalement, les patrons s'en remirent à la décision de 
Mgr L.-Nazaire Bégin, archevêque de Québec, et les travailleurs s'empressèrent de les imiter, ceux-là promettant de rouvrir leurs portes, et ceux-ci, de ne pas se réunir pour discuter de la question avant le prononcé de la sentence.

Mgr Bégin fit connaître sa décision le 14 janvier 1901. «Le droit de se constituer en association de métiers, de professions, d'emplois quelconques », y est-il écrit, " est un droit naturel; il a toujours existé et il existera toujours. Mais de ce que ce droit ne peut être méconnu, il ne s'ensuit pas que toutes les associations soient légitimes. Pour qu'elles aient droit à l'existence et puissent faire du bien, il faut qu'elles se proposent d'atteindre une fin honnête et juste et qu'elles n'emploient, pour y arriver, que des moyens conformes à la morale, à l'honnêteté et à la justice...

«J'ai examiné soigneusement les Constitutions et Règlements de la Fraternité des Cordonniers-Unis, de l'Union protectrice des Cordonniers-Monteurs, et de la Fraternité des Tailleurs de cuir; je ne saurais les approuver sans qu'ils aient subi un certain nombre de modifications ....

On sait que les travailleurs concernés tinrent compte des avis de l'archevêque de Québec, modifièrent leurs constitutions et dans la suite demandèrent et obtinrent des aumôniers.

En second lieu, l'éminent arbitre proposa aux parties une formule de conciliation et d'arbitrage, «pour tout litige qui pourrait se présenter à l'avenir ». Les ouvriers devaient constituer un «Comité de Réclamation» de trois membres, et les patrons, un «Comité de Conciliation» également de trois membres.

Quand un ouvrier aura quelque plainte à faire contre son patron, il la formulera par écrit, la fera signer par deux de ses compagnons de travail et la communiquera au «Comité de Réclamation», avec prière de la transmettre au «Comité de Conciliation 》. Les membres de ces deux comités examineront conjointement le cas à résoudre, chercheront à amener une entente et, dans le cas où l'entente serait impossible, le «Comité de Réclamation 》 portera la plainte immédiatement devant le « Tribunal d'Arbitrage ».

Quand la plainte viendra directement de la part d'un manufacturier, celui-ci s'adressera directement au «Comité de Conciliation 》, qui informera de suite le «Comité de Réclamation 》 du grief du patron, afin de venir à une entente, puis, si elle n'a pas lieu, le «Comité de Conciliation» aura recours au «Tribunal d'Arbitrage \$. (Texte de la sentence).

Si les parties ne parviennent pas à s'entendre, le litige devra être tranché par un tribunal d'arbitrage formé de trois membres: le premier, nommé par les ouvriers, le second, délégué par les patrons, et le troisième, choisi par ces deux premiers arbitres, et sinon, par un juge de la Cour Supérieure ou par l'archevêque de Québec. Le tribunal n'aura qu'une durée d'un an, et les mêmes membres sont rééligibles. La sen- 
tence arbitrale est exécutoire. Durant la constitution de la preuve, pas de grève ni de contre-grève.

C'était la première fois dans la province de Québec et au Canada qu'un archevêque catholique réglait un conflit ouvrier. Inutile de dire que l'arbitrage de Mgr Bégin dans l'industrie de la chaussure exerça une forte influence sur notre cabinet provincial, influence qui se manifesta par l'adoption, en 1901, de la Loi des différends ouvriers de Québec.

Les unions internationales, alors dominantes dans la Province, ne virent pas d'un très bon oeil la part prépondérante prise par Mgr Bégin dans la solution de ce conflit. Leurs chefs redoutaient l'ingérance du clergé dans les associations ouvrières. C'est pourquoi, en dépit de la défiance marquée de tous les syndiqués de l'époque à l'endroit des contraintes légales et des interventions gouvernementales, ils firent pression auprès des pouvoirs publics pour obtenir un organisme étatique de conciliation et d'arbitrage. L'hon. Lomer Gouin prit sur lui de les satisfaire.

\section{ANALYSE DE LA LOI DES DIFFÉRENDS OUVRIERS (1901)}

La Loi des différends ouvriers de Québec a sa source dans la loi néozélandaise de 1894, amendée en 1896 et 1898, et finalement refondue en 1900 sous le titre de "Industrial Conciliation and Arbitration Act». L'influence de cette loi sur la nôtre se fait surtout sentir dans la constitution des tribunaux d'arbitrage. Mais notons sur-le-champ une différence fondamentale dans l'esprit des deux lois: celle de la NouvelleZélande imposait le recours à l'arbitrage, alors que notre loi, à l'instar de la loi fédérale de 1900 et de la loi française de 1892, laisse toute liberté d'utiliser ou non la procédure de conciliation et d'arbitrage. Il faudra attendre la Loi des relations ouvrières (1944) pour assister à une modification radicale de cette conception.

La loi s'applique à toute industrie qui emploie au moins dix ouvriers, et à tout différend portant sur les salaires, les heures de travail, l'exécution d'une convention, la qualité du travail, les mauvais traitements aux employés, l'insalubrité des locaux et "le renvoi d'employés à raison de leur affiliation à une asociation ouvrière »

Le « greffier » nommé en vertu de la loi doit enregistrer les demandes de conciliation et d'arbitrage, convoquer les conseils en cause pour étude et jugement, consigner par écrit les détails afférents aux différends soumis, assigner au besoin des témoins et donner son avis. L'influence de la loi française de 1892 se fait ici fortement sentir.

Le conseil de conciliation chargé de prendre connaisance d'un litige se composera de quatre personnes: 2 pour les travailleurs et 2 pour les employeurs. Comme le système de conciliation est libre, un litige ne 
peut être soumis à un tel conseil que si les deux parties en demandent ensemble la formation, ou si l'une ou l'autre des parties fournit au greffier les détails du conflit. Après délibération, le conseil de conciliation remet au greffier un rapport écrit. A défaut d'entente, l'une ou l'autre des parties peut demander au greffier de porter le différend devant le conseil d'arbitrage.

La loi institue deux sortes de tribunaux d'arbitrage: l'un s'applique aux différends survenus dans les chemins de fer, et l'autre à tous les autres types d'entreprise. Chacun deux devra compter trois membres, sujets britanniques, nommés par le lieutenant-gouverneur en conseil: l'un proposé par la partie patronale, l'autre par la partie ouvrière, et le dernier suggéré par les deux premiers en-dedans de 21 jours, à défaut de quoi le lieutenant-gouverneur en conseil le désignera d'office. Une même personne pourra présider les deux conseils. Les membres sont nommés pour deux ans, et rééligibles. Les séances sont publiques. La sentence doit être rendue un mois au plus tard après la fin de l'instruction. Lorsqu'une des parties ou les deux n'accepteront pas la sentence arbitrale, elles pourront recourir à un nouveau conseil de conciliation. Les parties peuvent s'entendre au préalable pour accepter la sentence comme obligatoire. La mise à exécution de la loi relève du ministère nouvellement fusionné de la Colonisation et des Travaux publics, sous la direction de l'hon. Lomer Gouin (2 juillet). Il en sera ainsi jusqu'en 1905.

En somme, le législateur avait édifié un mécanisme fort méritoire, assez compliqué, mais dont l'usage était parfaitement facultatif. Dans un pareil domaine, et compte tenu de l'atttiude dominante d'alors à l'endroit des «ingérences de l'Etat», une loi aussi peu contraignante ne pouvait donner que de maigres résultats et s'en remettait trop facilement à la personnalité du greffier même. Nous aurons l'occasion, dans les pages qui vont suivre, d'assister à la marche du mécanisme.

\section{LES ANNÉES 1902-1905}

Dans son rapport de 1902, Louis Guyon, chef du service d'inspection depuis le 28 décembre 1900 , ne cache pas son contentement à la suite d'un amendement à la Loi des édifices publics qui soumet à l'inspection « les magasins de toutes dimensions, et les bâtiments de 3 étages ou plus au-dessus du rez-de-chaussée qui sont occupés comme bureaux ». (63 vict., ch. 22 , du 23 mars 1900 )

Félix Marois, premier greffier des conseils de conciliation et d'arbitrage écrit dans son rapport (1902):

Il est toujours regrettable de voir les différends entre le capital et le travail, au lieu de se résoudre promptement, se prolonger en chômage ruineux; cela n'est pas toutefois de nature à surprendre. Avec l'opinion actuelle que l'on a de l'arbitrage, chez la plupart des patrons comme chez un grand nombre d'ouvriers, on ne peut guère espérer de plus notables effets de cette institution qui, si elle était bien comprise, produirait sans aucun doute un bien considérable. 
La plupart du temps, en effet, c'est quand le conflit est entré dans sa phase aiguë que l'on s'efforce de concilier les deux parties: on essaye de prêcher la paix, quand déjà les armées sont rangées en bataille; on voudrait obtenir des concessions, des transactions, des accommodements de la part d'ennemis tout prêts à se livrer combat. L'arbitrage dans ces conditions devient très difficile. On ne doit pas l'oublier, l'arbitrage est beaucoup plus propre à prévenir les conflits qu'à les arrêter lorsqu'ils sont devenus violents et que l'aigreur a fermenté dans les esprits (p. 183).

\section{Quel remède apporter? Le greffier offre la solution suivante:}

La loi ne contient aucune disposition admettant l'intervention spontanée d'un fonctionnaire du gouvernement, pour aider au règlement de difficultés en existence. Un amendement devrait être rédigé de manière à pourvoir à des moyens d'exécution moins compliqués, en vue d'atteindre le but visé dans la loi. L'on atteindrait ce but en donnant, dans certaines circonstances, au greffier des conseils de conciliation et d'arbitrage le droit de se rendre lui-même dans la localité où une grève ou une fermeture de fabrique est appréhendée ou existe, afin de prêter ses bons services, en vue d'arriver à un règlement de la difficulté en cherchant à calmer les défiances, écarter les causes de friction, favoriser le bon vouloir... et les conventions entre patrons et employés, dans le but de les amener à soumettre leurs différends à un tribunal de conciliation ou d'arbitrage avant de recourir aux grèves ou à la fermeture des ateliers (p. 184).

Les voeux de M. Marois allaient être exaucés dès l'année suivante (25 avril 1903) par l'insertion de l'article 6A dans la loi de 1901: s'il y a différend ou menace de différend, le greffier doit, à la demande par écrit du patron, ou de cinq employés, ou du maire, ou simplement en apprenant la nouvelle, se rendre sur les lieux et tenter de concilier les parties, de les amener à soumettre leur différend à un conseil de conciliation ou d'arbitrage. C'est le 19 novembre 1902 seulement que la Loi des différends ouvriers a servi pour la première fois. Les rapports des inspecteurs en 1903 sont fort intéressants. Louis Guyon souligne l'adoption, au début de l'année, d'un amendement à la Loi des établissements industriels qui empêche dans tous les cas les garçons de moins de 13 ans (non plus 12) de travailler. Selon lui, «tout accuse une ère de prospérité et d'activité manufacturières sans précédent dans le pays » (p. 170). Mme Provencher nous fait part de nouveaux règlements qui obligent les jeunes filles à porter des tresses pour éviter les accidents, les employeurs à fournir des sièges aux femmes et des crachoirs aux hommes.

Mme King, inspectrice dévouée, est aussi une humoriste hors pair. Lisons:

Comme la rosée bienfaisante qui tombe sans bruit sur les plantes altérées et les fait reverdir; comme les rayons du soleil qui répandent la joie et la santé partout où ils pénètrent, de même l'inspectrice accomplit sa mission... (p. 187).

Plus loin elle s'en prend aux «femmes dont la taille ressemble plus à un sablier qu'au torse de la Vénus de Milo »!

P.-J. Jobin, inspecteur à Québec, rend le témoignage suivant:

Les moulins de pâte de bois fournissent un travail continu de 24 
heures par jour. Les hommes sont divisés en deux équipes, l'une travaillant le jour pendant 11 heures, l'autre travaillant la nuit pendans 13 heures (p. 193).

$\mathrm{Au}$ cours de toutes ces années, le greffier des conseils de conciliation et d'arbitrage fait plutôt office de «rapporteur» des conflits industriels en cours. On ne sent que rarement, dans la description qu'il en fait, le poids de son intervention. Sa position est particulièrement difficile en raison même du texte et de l'esprit de la loi. Il reste que les commentaires nombreux qu'il fait sur les litiges du temps sont fort utiles et révélateurs.

Et c'est ainsi que nous en arrivons à l'année 1906, qui assiste à l'adjonction expresse d'une section du travail au Ministere des travaux publics, qui a nom désormais Ministère des travaux publics et du travail (3 juillet). Le premier en date des ministres est l'hon. L.-J. Allard, qui sera remplacé dès l'année suivante par l'hon. W. A. Weir; les services d'inspection et de conciliatíon relève dorénavant de ce ministère, jusqu'en 1931.

\section{LES ANNÉES 1906-1908}

C'est en 1906 que le Parlement fédéral vota la Loi du dimanche, sur les instances du travail organisé, de la Ligue du dimanche et d'un comité parlementaire spécialement chargé d'étudier la question. Cette loi interdit la vente d'articles et l'emploi de personnes dans les établissements industriels et commerciaux le dimanche, exception faite des «travaux d'urgence ou d'humanité ». Dès l'année suivante, les députés du Québec adaptaient la Loi concernnat l'observance du dimanche, qui fut sanctionnée le 28 février 1907 ( 7 Ed. VII, ch. 42). Aux termes de la nouvelle loi, «Il est défendu, le dimanche, dans un but de lucre, sauf néanmoins le cas de nécessité ou d'urgence, d'exécuter ou de faire exécuter aucune oeuvre industrielle, ainsi que d'exercer aucun négoce ou métier ou de donner ou d'organiser des représentations théâtrales, ou de sexcursions accompagnées de vente de liqueurs enivrantes, ou de prendre part ou d'assister à ces représentations théâtrales ou à ces excursions » (article 2). La Loi provinciale venait régler la question de juridiction qu'avait pu soulever la loi fédérale de l'année précédente.

En 1906 toujours, la Loi de la conciliation et du travail fédérale unifie la Loi de la conciliation de 1900 et la Loi relative aux difficultés ouvrières dans les chemins de fer (1903). Cette année-là, le service d'inspection compte cinq hommes et deux femmes, depuis 1894, le budget du bureau n'a pas changé. Les inspecteurs des chaudières font près de 2,500 visites. Vingt-trois accidents sont rapportés qui ont des suites fatales, sur 485 en tout. C'est dans le rapport de cette année qu'est décrite la grève de 125 tailleurs de cuir dans 10 fabriques de Montréal, qui se termina par l'intervention arbitrale de Mgr Paul Bruchési, archevêque de Montréal. 
L'année 1907 voit l'adoption d'un amendement très important à la Loi des établissements industriels provinciale ( 7 Ed. VII, ch. 39). A l'avenir, même dans les établissements non classés comme dangereux, l'âge de tout enfant ne devra jamais être inférieur à 14 (non plus 13) ans; et l'inspecteur aura le droit d'exiger que le certificat d'âge soit vérifié par affidavit. Un nouvel article (3024A), aux répercussions sociales considérables, se lit comme suit:

Tout garçon et toute jeune fille au-dessous de seize ans employés dans un établissement industriel et qui ne savent ni lire ni écrire doivent, tant qu'ils continuent d'être ainsi employés, ou jusqu'à ce qu'ils sachent lire et écrire, fréquenter continuellement une école du soir de la municipalité où ils résident, s'il y en a une, et aucun patron ne doit admettre de jeune garçon ou de jeune fille dans son établissement sans s'être assuré que ce jeune garçon ou cette jeune fille sait lire et écrire, ou (suivant le cas) sans un certificat du directeur ou autre instituteur en charge de cette école du soir attestant que ce jeune garçon ou cette jeune fille fréquente la dite école. Ce certificat doit être conservé dans l'établissement, et montré à l'inspecteur chaque fois qu'il en fait la demande.

Louis Guyon, commentant cet amendement, écrit dans san rapport: « Notre législation, en prescrivant que tout garçon ou toute jeune fille de 14 à 16 ans devrait désormais savoir lire et écrire avant d'entrer à la fabrique, ou tout au moins se préparer à satisfaire aux nouvelles exigences de la loi par la fréquentation d'une école du soir, nous a d'un seul coup placés au niveau intellectuel des autres pays en matière de législation ouvrière». Il rappelle aussi que le service d'inspection possède « une bonne bibliothèque technique ouverte à tous ». Le nouvel inspecteur O.-J. Monday, de Montréal, raconte une mésaventure qui indique bien le peu de cas qu'on faisait en certains milieux de l'autorité de l'inspecteur du travail:

Dans un établissement, j'ai été même éconduit par la force. Le patron me prit par le bras et me poussa vers la porte de sortie. Comme je protestais en disant que j'avais un devoir à remplir comme inspecteur envoyé par la loi, il me donna force coups de pieds et coups de poings jusqu'à ce que je fusse hors de l'établissement (p. 111).

Il est consolant de se dire que de pareils gestes ne seraient pas posés de nos jours sans qu'il en coûte très cher à leurs auteurs!

Dans le cas des chaudières à vapeur, l'arrêté 183 du 28 mars 1907 casse l'exception consentie à l'article 31 des «Règlements 》 de 1894, en faveur des beurreries et fromageries, et statue que "l'inspection des chaudières à vapeur et conduites-vapeur et moteurs, dans les fabriques de beurre et de fromage, ne sera obligatoire que tous les deux ans . . . » L'arrêté 231 du 26 avril suivant divise la province en district d'inspection, fixe le prix des inspections, les types des certificats à décerner, et oblige à l'inspection annuelle.

C'est en 1907 également que le Parlement fédéral vote la Loi des enquêtes en matière de différends industriels, dite «Loi Lemieux 》 (6-7, Ed. VII, ch. 20). L'année précédente avait été témoin de plusieurs trou- 
bles industriels sérieux, notamment dans une scierie de Buckingham (Québec) et dans les tramways à Hamilton et Winnipeg; une grève de neuf mois dans les charbonnages de l'Alberta mit le comble. La loi fut adoptée à la session suivante. Elle couvrait les services publics. Elle obligeait à la tenue obligatoire d'une enquête par une commission de conciliation nommée par le gouvernement; elle prohibait les arrêts de travail avant la tenue de l'enquête; elle donnait à une opinion publique éclairée l'occasion d'influer sur les jestes des parties en présence. On contesta la légalité de cette loi en 1923 dans un conflit impliquant la Toronto Electric Company et ses employés. L'affaire fut finalement portée au Conseil Privé, qui en 1925 déclara la loi inconstitutionnelle et donc invalide; la décision précisa qu'en matière de travail, l'autorité appartient directement aux provinces, et qu'en général la grève n'est nullement dangereuse pour la paix, l'ordre et le bon gouvernement du pays sauf en cas de guerre, naturellmeent. Quoi qu'il en soit de cette décision, le Parlement de Québec, à la demande du Congrès des Métiers et du Travail et à l'instar de celui de l'Ontario, s'empressa en 1932 (22 Geo. V, ch. 46) de revalider la Loi fédérale dans les limites de la Province et pour les cas où elle s'appliquait.

L'hon. L.-A. Taschereau succède à l'hon. W.-A. Weir, le 17 octobre 1907 comme ministre des Travaux publics et du Travail. Il occupera ce poste, toujours sous le gouvernement Gouin, jusqu'en 1919, contribuant ainsi à assurer aux services que nous étudions une administration stable. L'année suivante, et durant seize ans, il sera premier ministre de la Province.

En 1908, la Loi des édifices publics est complètement refondue et améliorée ( $8 \mathrm{Ed}$. VII, ch. 52). Plusieurs nouvelles catégories d'édifices sont couverts - monastères, tous les hôpitaux, orphelinats, crèches, édifices d'exposition ou d'amusement, magasins de 10 commis ou plus, palais de justice, etc. Le système de sauvetage doit être approuvé par linspecteur. Des tragédies récentes justifient de nombreux articles pour la prévention des incendies et des paniques dans les salles de représentation, les collèges et les hôtels.

Une nouvelle Loi concernant l'inspection des échafaudages (8 Ed. VII, ch. 53) s'inscrit le 25 avril 1908 dans les statuts. Toute cité ou ville peut nommer et rémunérer des inspecteurs. Pour les échafaudages d'au moins 15 pieds de hauteur, l'entrepreneur doit obtenir et exhiber un certificat d'inspection fourni par un inspecteur, un ingénieur ou un architecte. Les inspecteurs ont toute autorité pour examiner, et au besoin condamner les échafaudages. Le ministre des Travaux publics et du Travail est responsable de la mise à exécution de la Loi.

\section{Dans son rapport de 1908, Louis Guyon s'exprime ainsi:}

L'obligation pour les enfants employés dans les fabriques entre les âges de 14 et 16 ans de savoir lire ou écrire ou de fréquenter une école du soir est très difficile à satisfaire pour les enfants d'abord, parce que dans bien des cas, il n'existe pas d'écoles du soir, ou tout 
au plus en existe-t-il pour les garçons. D'un autre côté, il est presque impossible pour les patrons de contrôler efficacement la fréquentation assidue des classes par les jeunes employés visés par le règlement. Cette surveillance déléguée aux inspecteurs constitue une charge bien lourde pour eux (p. 91).

\section{CONCLUSION SUR CETTE PÉRIODE}

Tous les rapports des inspecteurs et du greffier, au cours de la première décennie de ce siècle, attestent une poussée extraordinaire de l'industrialisation dans la province de Québec. Les établissements se multiplient et prennent de l'ampleur. L'exode des campagnes vers les villes devient plus apparent. Les agglomérations de travailleurs déracinés, dans les usines et dans les villes, créent des circonstances nouvelles qui se traduisent par un esprit nouveau. Les salaires restent bas, la sécurité financière est un mot. Les travailleurs, pour améliorer leurs conditions de travail et assurer leur sécurité, se groupent toujours plus nombreux dans les syndicats. Des grèves dures et prolongées, lourdes de misères nombreuses et de ressentiments explosifs, viennent ajouter à la tension industrielle. La législation assez lâche du temps enlève beaucoup d'efficacité au travail du greffier des conseils de conciliation et d'arbitrage.

Dans le domaine de la protection des travailleurs et de l'assainissement des lieux de travail, l'effort des inspecteurs est fructueux. Une « coutume» s'est établie, et les conditions affreusement malsaines du début ne se retrouveront plus, au moins dans l'ensemble. On a tâché jusqu'ici de prévenir les accidents et les maladies. Dans un tel domaine, avec tous les risques présentés par l'industrie et les imprudences des travailleurs, le succès ne peut être complet. Il faudra donc prendre des mesures pour guérir de tels cas: nous les étudierons dans le chapitre suivant. 